\title{
Um sistema para auxílio no monitoramento da febre amarela
}

\author{
Caio A. N. Araujo ${ }^{1}$, Leila Silva ${ }^{1}$, Roseli La Corte ${ }^{2}$, Francielma S. Bittencourt ${ }^{2}$ \\ ${ }^{1}$ Departamento de Computação - Universidade Federal de Sergipe (UFS) \\ CEP 49.100-000 - Jardim Rosa Elze - São Cristóvão - SE - Brasil \\ ${ }^{2}$ Departmento de Morfologia - Universidade Federal de Sergipe (UFS) \\ CEP 49.100-000 - Jardim Rosa Elze - São Cristóvão - SE - Brasil \\ \{leila, roseli\}@ufs.br,$\{$ caioalexandre_144,francielmabittencourt\}@hotmail.com
}

\begin{abstract}
Yellowfever is an arthropode borne disease, with a worrying increase in the number of cases in Brazil. This work presents a tool to assist on the monitoring of the yellow fever transmitting agent. The tool was developed as part of a project to track the epidemiological situation of the state of Sergipe and aims to provide automatic support for the activities of capturing, manipulating, integrating and visualizing data collected by the team of this project. The system was developed following the Scrum methodology and can be accessed on the Web and mobile platforms.
\end{abstract}

Resumo. A febre amarela é uma doença transmitida por vetores, com crescimento preocupante de número de casos no Brasil. Este trabalho apresenta uma ferramenta para auxiliar no monitoramento dos agentes transmissores da febre amarela. A ferramenta foi desenvolvida como parte de um projeto de rastreamento da situação epidemiológica do estado de Sergipe e visa prover suporte automático às atividades de captura, manipulação, integração e visualização de dados coletados pela equipe desse projeto. O sistema foi desenvolvido seguindo a metodologia Scrum e pode ser acessado nas plataformas Web e móvel.

\section{Introdução}

A febre amarela é uma doença infecciosa febril aguda, causada por um arbovírus do gênero Flavivírus que tem como principais agentes transmissores os mosquitos dos gêneros Aedes e Haemagogus [OMS 2019]. Ela possui dois ciclos de transmissão: silvestre, quando há transmissão em área rural ou de floresta, e urbano. A febre amarela foi controlada no Brasil, após a efetivação de vários programas de combate à incidência desta doença, porém, nos últimos anos o número das notificações relacionadas ao vírus tem aumentado de maneira alarmante. Diante das ameaças de ressurgência do vírus da febre amarela em diversos estados brasileiros, é imprescindível o desenvolvimento de ações para monitorar os avanços nas áreas suscetíveis.

Os últimos casos registrados de febre amarela em áreas urbanas datam de 1942, porém o risco da ocorrência da doença em cidades está sempre iminente, devido à presença do vírus no ciclo silvestre, o qual tem apresentado epidemias cíclicas em períodos variados de 5 a 7 anos e à ampla infestação domiciliar por Aedes aegypti. Por isso, faz-se necessário a melhoria no monitoramento e controle dos vetores responsáveis e hospedeiros desses vírus em áreas silvestres e seu entorno. 
Com a ocorrência dos surtos de febre amarela em 2016 e 2017, em vários estados brasileiros, a atenção dos órgãos de saúde voltou-se para a doença após um período de poucas ações para o controle efetivo desta. Nesses anos vários estados se mobilizaram em campanhas de vacinação para evitar com que a doença pudesse trazer fatalidades à população [MS 2017]. Atividades de prevenção, associadas a campanhas de imunização podem ajudar sobremaneira no controle da doença. Entretanto, para realizar da melhor forma processos de controle e monitoramento na atualidade, é imprescindível o uso de tecnologia.

Este é o contexto do presente trabalho, que se insere no âmbito do projeto do monitoramento da febre amarela no estado de Sergipe. O projeto visa verificar a receptividade das Unidades de Conservação (UC) ao vírus da febre amarela, e contribuir diretamente para os esforços de vigilância em saúde no estado. O projeto compreende a análise entomoepidemiológica a partir de dados disponibilizados pelos órgãos de saúde, o desenvolvimento de atividades em campo nas áreas primárias de ocorrência e bordas das UC e a análise laboratorial do material coletado. A ferramenta proposta pretende contribuir para a agilidade no processamento de dados relacionados ao monitoramento da febre amarela, criação de um banco com as informações coletadas pelos pesquisadores e também com as informações das bases de dados históricos. Além disso, provê suporte à automação das notificações de alertas que podem auxiliar na tomada de decisão, controle e detecção dos possíveis surtos.

Dentre os trabalhos que associam o uso de tecnologia ao controle da febre amarela ou ao monitoramento do seu principal vetor em áreas urbanas, o mosquito Aedes aegypti, estão o Sistema de Informação de Agravos de Notificação [MS 1998], que objetiva a realização do diagnóstico dinâmico da ocorrência de um evento na população, podendo fornecer subsídios para explicações causais dos agravos de notificação compulsória; o trabalho proposto em [Dialynas et al. 2009] para monitoramento epidemiológico da resistência a inseticidas em mosquitos vetores de doenças; o sistema InfoDengue [FIOCRUZ 2015] que a partir da coleta de dados climáticos e relatos de dengue calcula a probabilidade de notificações ocorrerem; o trabalho proposto em [Morais et al. 2015] que apresenta uma abordagem computadorizada através de um mecanismo de interação hardware/software capaz de capturar imagens microscópicas de palhetas coletadas de ovitrampas e processar essas imagens realizando a contagem automatizada de ovos na amostra; e o trabalho publicado em [Gradella et al. 2016] que propõe um aplicativo móvel com uma abordagem diferenciada para coletar dados da população utilizando gamificação.

\section{Fundamentação Teórica}

\subsection{Monitoramento do vírus da febre amarela nas áreas suscetíveis}

O projeto no qual a ferramenta aqui descrita se insere visa obter resultados sobre a suscetibilidade ao vírus da febre amarela, relacionando os dados sobre as espécies de vetores e Primatas Não Humanos (PNH) presentes nas áreas selecionadas para monitoramento. As ações planejadas são: a pesquisa documental, desenvolvida com base em informações da febre amarela obtidas das fontes de estudo e de dados disponibilizados por órgãos de saúde; as pesquisas de campo, nas quais são realizadas coletas para levantamento de dados relativos às espécies existentes nas matas estudadas; e as pesquisas laboratoriais, que realizam a verificação de incidência de arboviroses e dados quantitativos de amos- 
tras coletadas. A ferramenta descrita neste artigo provê apoio computacional na coleta, armazenamento, correlação e visualização dos dados gerados por este projeto.

A pesquisa documental está relacionada com o levantamento dos dados referentes ao vírus, que irão contribuir para realizar os mapeamentos dos dados históricos determinados por: epizootias do estado de Sergipe nos últimos 10 anos, espécies de primatas e corredores ecológicos e das espécies de vetores.

As pesquisas de campo auxiliam no levantamento de dados das espécies relacionadas à transmissão da febre amarela. Para isso, são realizadas atividade de coleta de ovos, larvas e pupas, e a captura de adultos em localidades específicas, para obter informações necessárias para o monitoramento do vírus. As áreas de estudo elencadas têm regiões com incidência da Mata Atlântica, locais onde há a maior probabilidade de instalação do vírus, considerando a presença de primatas não humanos e disponibilidade de criadouros para os vetores silvestres. Essas áreas representam diferentes reservas e unidades de conservação, selecionadas por apresentarem características de interesse da vigilância contra a propagação do vírus.

As pesquisas de laboratório auxiliam na identificação das formas imaturas capturadas em campo, que são montadas em lâminas. Os imaturos são mantidos, em ambiente controlado, até emergir para a fase adulta. As formas imaturas e adultas são identificados em conformidade com as chaves de [Lane and Cerqueira 1942], [Correa and Ramalho 1956], [Forattini 1965, Forattini 2002], [Consoli and Lourenço-de Oliveira 1994] e outros trabalhos publicados por grupos de pesquisa na área. Os mosquitos encaminhados para análise de infecção são investigados por identificação taxonômica de acordo com [Reinert 2001].

O fluxo típico de atividades realizadas pela equipe de saúde do projeto inicia com o coordenador do projeto selecionando as atividades de campo a serem realizadas e estabelecendo as equipes e datas de coleta. Em seguida, os pesquisadores de campo vão para as áreas determinadas para realizar a implantação e manutenção das armadilhas já existentes. Estes pesquisadores realizam a coleta de amostras nas armadilhas de ovitrampas e de bambus. Nestas armadilhas, amostras são coletadas nas alturas $1 \mathrm{~m}$ e $3 \mathrm{~m}$ em armadilhas de ovitrampas e $1 \mathrm{~m}, 2 \mathrm{~m}, 3 \mathrm{~m}$, e $4 \mathrm{~m}$ nas de bambu. Após a coleta, as amostras são enviadas ao laboratório, onde os pesquisadores de laboratório fazem a verificação das espécies de mosquito existentes nas amostras, a emergência das palhetas coletadas com ovos e a análise da presença do vírus da febre amarela nos mosquitos.

Prover suporte computacional para o desenvolvimento das pesquisas de campo e laboratoriais deste projeto é o objetivo da ferramenta aqui apresentada. Para o entendimento do desenvolvimento do sistema proposto, as seções a seguir trazem uma visão geral da metodologia de desenvolvimento utilizada, linguagens e tecnologias adotadas.

\subsection{Framework Scrum}

Neste trabalho adotou-se a metodologia Scrum, adaptada à existência de apenas um desenvolvedor. O ponto de partida do fluxo é a confecção do Backlog do Produto, que é o artefato que dá suporte ao armazenamento e gerenciamento dos requisitos coletados. Em seguida, várias iterações, chamadas Sprints, são realizadas até a obtenção do produto final, como pode ser visualizado na Figura 1. 


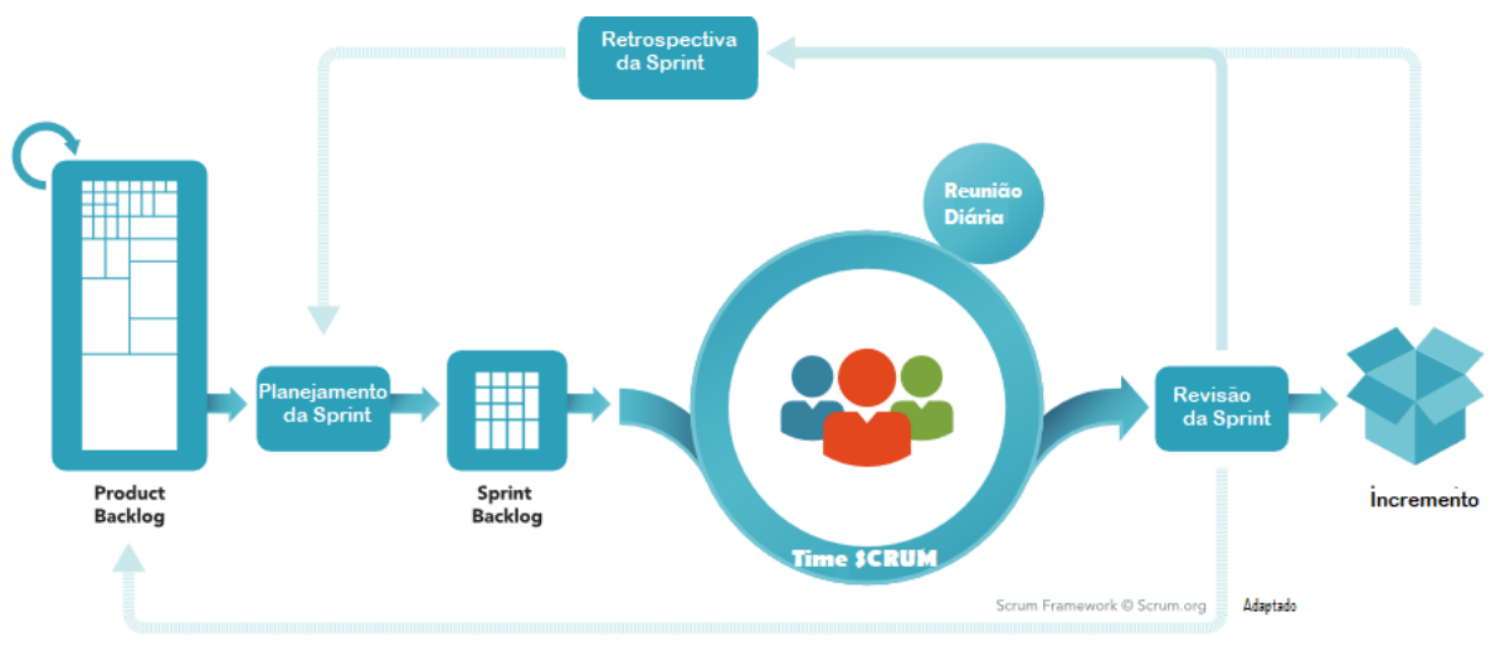

Figura 1. Fluxo Típico do Scrum (extraído de Scrum.org ${ }^{1}$ ).

Os papéis estabelecidos no Scrum são Product Owner (PO), Time de Desenvolvimento e o Scrum Master (SM). O PO é o responsável por maximizar o valor do produto final, e o encarregado pelo Backlog do Produto, que é fundamental para validar o andamento da produção do produto. O Time de Desenvolvimento é composto pelos profissionais que irão incrementar o produto a cada Sprint. O SM é responsável por prover suporte e promover o Scrum, gerenciando todo o processo.

O produto é desenvolvido em Sprints. Os eventos Scrum são: o Planejamento da Sprint em que o trabalho a ser realizado é planejado, a Reunião Diária para alinhar o andamento do projeto e planejar o próximo dia, a Revisão da Sprint realizada ao fim do ciclo objetivando verificar a melhoria incrementada e a Retrospectiva da Sprint que contempla uma inspeção do time para tratar ajustes e melhorias dos membros. Todas as Sprints são time-boxed, ou seja, possuem duração fixada.

Os artefatos Scrum são: o Backlog do Produto que é uma lista de requisitos desejadas pelo PO para o produto final, Backlog da Sprint é uma parte do Backlog do Produto que será trabalhada na iteração vigente, e o Incremento que é resultado dos esforços do time de desenvolvimento durante a iteração trabalhada. Ao fim de cada Sprint inicia-se a próxima Sprint, e assim continua em ciclo, até que o produto final seja feito.

\subsection{Linguagens, Ferramentas e Tecnologias utilizadas}

A apresentação de algumas informações georreferenciadas foi feita com o uso da API ArcGIS JavaScript ${ }^{2}$ que é integrada ao JSF por meio da a biblioteca GISFaces ${ }^{3}$, auxiliando a apresentação de mapas em aplicações, mais intuitiva e facilitada. Complementando o JSF, foi utilizada a biblioteca PrimeFaces ${ }^{4}$. A ferramenta foi desenvolvida com IntelliJ IDEA $2019.1^{5}$ que é um Ambiente de Desenvolvimento Integrado (em inglês, Integrated

\footnotetext{
${ }^{1}$ https://www.scrum.org/resources/what-is-scrum

${ }^{2}$ https://developers.arcgis.com/javascript/

${ }^{3}$ https://www.gisfaces.com/

${ }^{4}$ https://www.primefaces.org/

${ }^{5}$ https://www.jetbrains.com/idea/
} 
Development Environment (IDE)) completo para a linguagem Java. A apresentação em dispositivos móveis foi confeccionada com Flutter ${ }^{6}$, o qual permite o desenvolvimento para Android, iOS e Web, utilizando um só código.

\section{A ferramenta proposta: SIMFA}

De acordo com a metodologia Scrum, inicialmente o Backlog do produto foi construído. Para isso, fez-se necessário proceder ao levantamento dos requisitos do sistema. Para expressar os requisitos levantados foi desenvolvido o diagrama de casos de uso, adotandose a Linguagem de Modelagem Unificada (UML) [Fowler 2005].

O sistema prevê quatro tipos de atores: Visitante, Pesquisador Campo, Pesquisador Laboratório e Coordenador. O ator Visitante é o usuário que não possui acesso à parte interna do sistema, ele pode acessar as páginas públicas e solicitar acesso ao sistema. O ator Pesquisador Campo é responsável pela realização das atividades de campo, que consistem na coleta das informações de armadilhas implantadas em diversas áreas de preservação. Cabe ao Pesquisador Campo inserir esses dados no sistema. O ator Pesquisador Laboratório irá complementar as informações inseridas pelos Pesquisadores de Campo, realizando os procedimentos em laboratório, introduzindo os resultados no sistema. O ator Coordenador, além de ser o usuário responsável pela gestão do sistema, atuando na autorização de usuários novos, solicitação de novas atividades e criação de equipes, pode realizar as mesmas funções atribuídas aos pesquisadores. A Figura 2 mostra o diagrama de casos de uso da ferramenta, onde a notação de bonecos simbolizam os atores do sistema e as elipses os casos de uso do sistema.

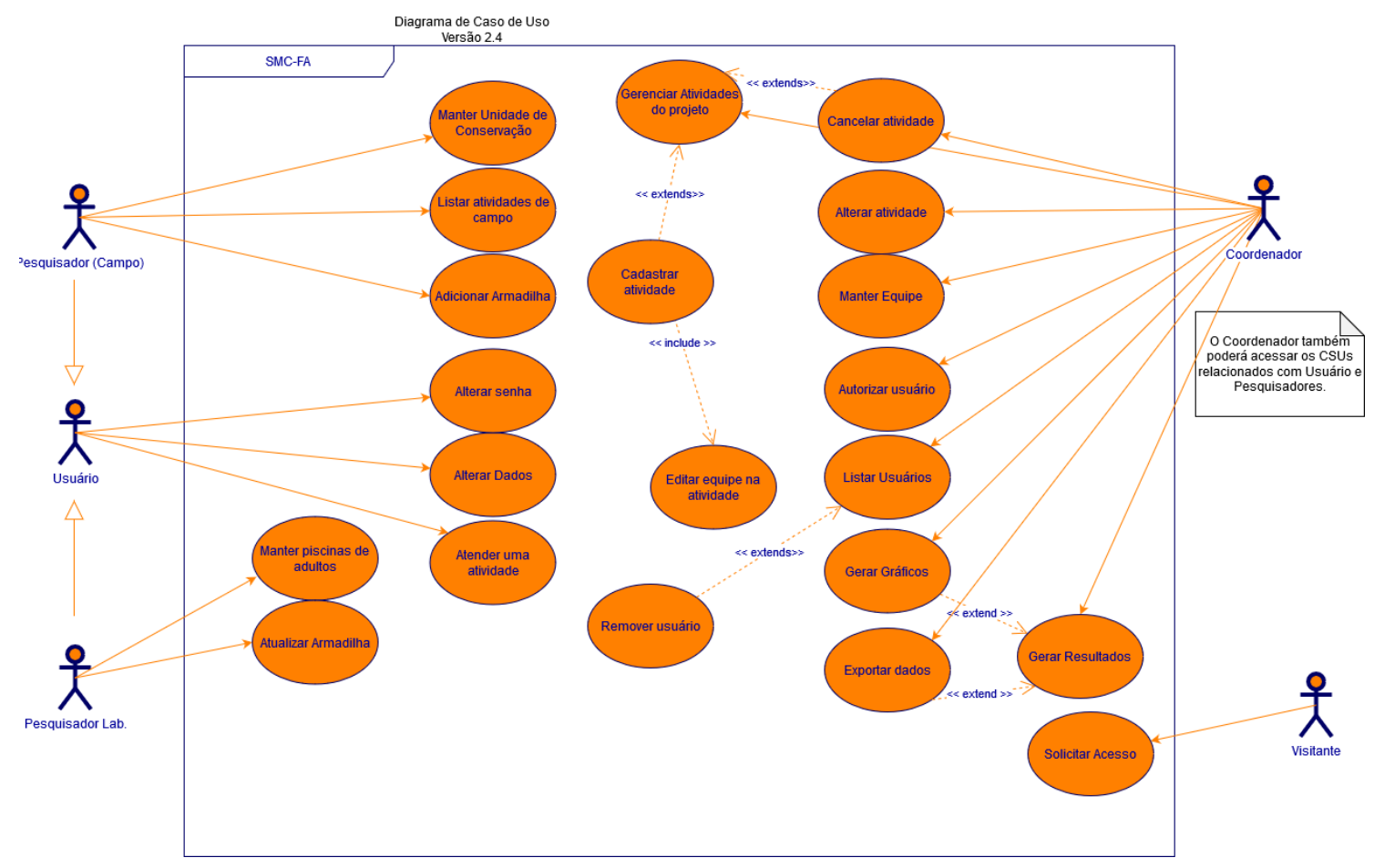

Figura 2. Diagrama de Caso de Uso

\footnotetext{
${ }^{6}$ https://flutter.dev/
} 


\subsection{Backlog do Produto}

Após o levantamento de requisitos chegou-se ao Backlog do produto mostrado na Figura 3. Neste artefato todas as funcionalidades do produto estão listadas, priorizadas com base nas necessidades do PO, que no caso foi a equipe da área de saúde deste projeto.

\begin{tabular}{|c|c|c|c|}
\hline \multicolumn{4}{|c|}{ BACKLOG DO PRODUTO SCRUM } \\
\hline ITEM \# & DESCRIÇÃO & PONTOS EST. & PRIORIDADE \\
\hline 1 & Projetar um protótipo & 5 & BAIXA \\
\hline- & Preparação do ambiente de desenvolvimento & - & - \\
\hline 2 & - Configuração da IDE para integração das tecnologias JavaEE & 2 & DEV \\
\hline 3 & - Configuração JSF + PrimeFaces (Front-end) & 3 & DEV \\
\hline 4 & - Configuração do Controller com CDI e Managed Beans & 3 & DEV \\
\hline 5 & - Configuração do GISFaces (API ArcGis) & 3 & DEV \\
\hline 6 & - Configuração JPA para as Regras de Negócio (Back-end) & 3 & DEV \\
\hline 7 & - Modelagem do banco de dados (JPQL) & 3 & DEV \\
\hline 8 & - Integração com o banco de dados Postgres & 3 & DEV \\
\hline- & Gerenciamento de unidades de conservação (UC) & & - \\
\hline 9 & - Desenvolvimento do CRUD de UCS & 5 & ALTA \\
\hline 10 & - Integração das informações da UC com Atividades e Armadilhas & 10 & ALTA \\
\hline- & Gerenciamento de atividades do projeto & & - \\
\hline 11 & - Desenvolvimento do CRUD de Atividades & 5 & ALTA \\
\hline 12 & - Integração das informações de Atividades e Equipes & 5 & MEDIA \\
\hline 13 & - Preparo de resultados da execução de atividades & 5 & BAIXA \\
\hline- & Gerenciamento de equipes & - & - \\
\hline 14 & - Integração das informações de Usuário e Equipes & 5 & MEDIA \\
\hline 15 & - Desenvolvimento do CRUD de Equipes & 5 & ALTA \\
\hline- & Gerenciamento de armadilhas & & - \\
\hline 16 & - Desenvolvimento do CRUD de Armadilhas & 5 & ALTA \\
\hline 17 & - Análise de implantação de armadilhas em UCs & 5 & ALTA \\
\hline 18 & - Atribuições das análises laboratóriais para as armadilhas & 8 & MEDIA \\
\hline 19 & Apresentação em Dispositivo Móvel & 5 & BAIXA \\
\hline 20 & Emissão de gráficos & 5 & MEDIA \\
\hline 21 & Emissão de relatórios & 3 & ALTA \\
\hline- & Exportação de dados & - & - \\
\hline 22 & $\begin{array}{l}\text { - Permitir exportação dos dados presentes nas UCs (Atividades, } \\
\text { Armadilhas) em formato tabulado (Excel ou CSV) }\end{array}$ & 2 & BAIXA \\
\hline- & Gerenciamento de Usuários & - & - \\
\hline 23 & - Configurações de acesso público ao sistema & 2 & MEDIA \\
\hline 24 & - Desenvolvimento do CRUD de Usuários & 3 & MEDIA \\
\hline 25 & - Configurações de acesso restrito às informações de Usuários & 2 & ALTA \\
\hline & PONTUAÇÃO TOTAL & 100 & - \\
\hline
\end{tabular}

Figura 3. Backlog do Produto

O sistema foi desenvolvido ao longo de sete Sprints, cada uma delas compreendeu o período de quinze dias contemplando um conjunto de requisitos do Backlog do produto para o desenvolvimento. As Sprints seguiram o processo Scrum, com planejamento da Sprint, definição do Backlog da Sprint, desenvolvimento do projeto, Revisão da Sprint e a Retrospectiva da Sprint. A Figura 4 mostra um fragmento do diagrama de classes obtido após a sétima Sprint, usando a notação UML. Neste diagrama estão representadas as principais classes do sistema, permitindo uma visualização estrutural da ferramenta.

As principais classes do sistema são: Perfil, Usuário, Equipe, Atividade de Campo, Unidade de Conservação, Armadilha, Bambu, Ovitrampa, Coletor de Adulto, Primata, Pool, Especie. As classes Perfil e Usuario são responsáveis pela verificação de acesso e auxiliam no controle de inserção de dados no sistema. Equipe compreende 
as equipes que executam as atividades previstas no projeto; Atividade de Campo possui os dados de atividades de campo executadas; Unidades de Conservação concentra as informações onde são executadas as ações de monitoramento; Armadilha é a classe abstrata que possui informação em comum dos tipos de armadilhas identificados, Bambu, Coletor de Adulto e Ovitrampa, as quais também têm dados específicos de informação coletada; Primata armazena as informações das espécies de primatas não humanos catalogadas, Pool compreende as informações dos ovos colocados para emergir; e Especie que é a classe de cada tipo de espécie de mosquito monitorada pelo projeto.

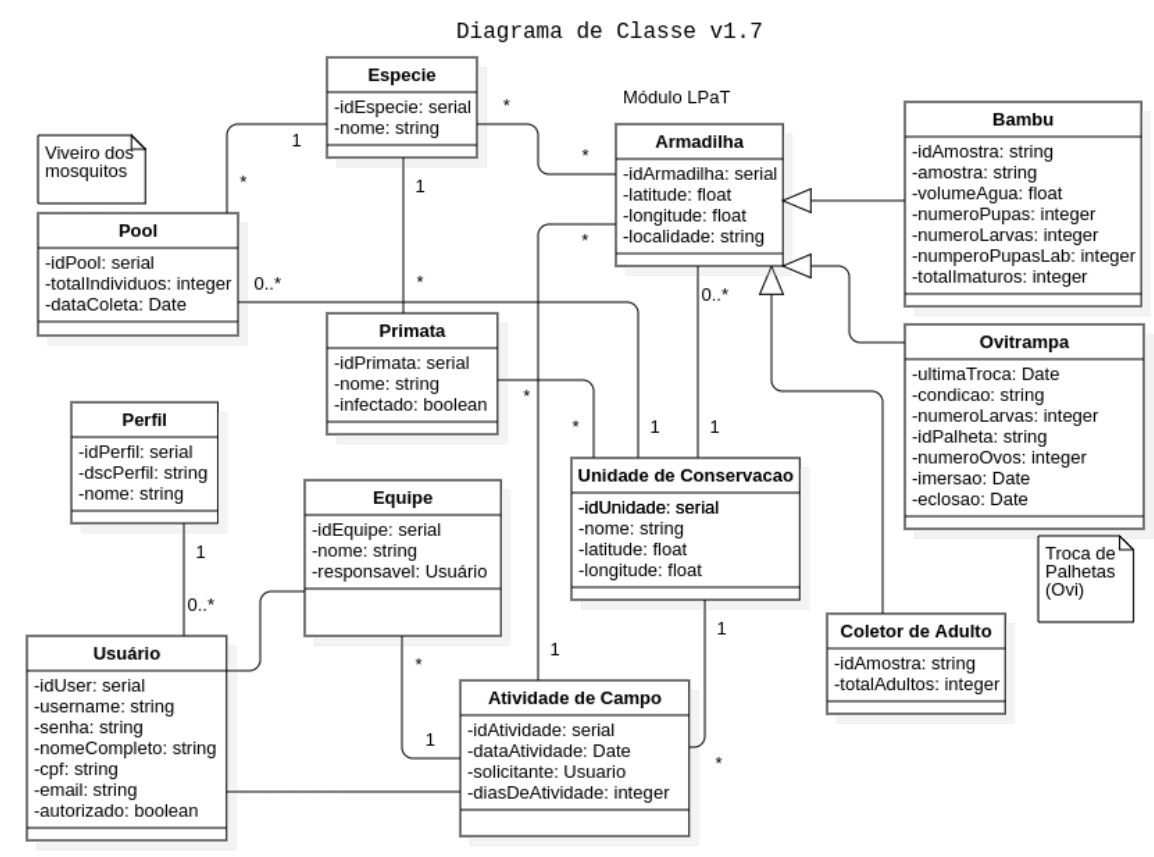

Figura 4. Fragmento do Diagrama de Classes

Um fluxo típico do uso da ferramenta é apresentado na Figura 5. O fluxo inicia-se com o acesso do usuário no sistema, o qual deve informar suas credenciais corretamente. O usuário tem então algumas opções disponíveis expressas nos subfluxos apresentados. Para o cadastro de informações, o usuário acessa o formulário correspondente ao tipo de informação que ele deseja inserir no sistema, preenche todos os dados necessários e confirma a operação de cadastramento dos dados. Após realizar o cadastro, ele pode novamente escolher outras opções disponibilizadas pelo sistema.

Para consultar informações, o usuário acessa no menu a parte de Consulta, e informa alguns parâmetros desejados na consulta, caso necessário. Após realizar essas etapas ele pode consultar os dados em tela. Para alguns tipos de consulta há também a possibilidade de emitir relatórios. Independente da operação escolhida, após a execução da operação, o usuário pode escolher entre realizar uma nova operação ou fazer o logout na ferramenta. Algumas funcionalidades como a exportação de dados, a autorização de acesso, e algumas partes da execução em dispositivos móveis não são contempladas por esse diagrama. 


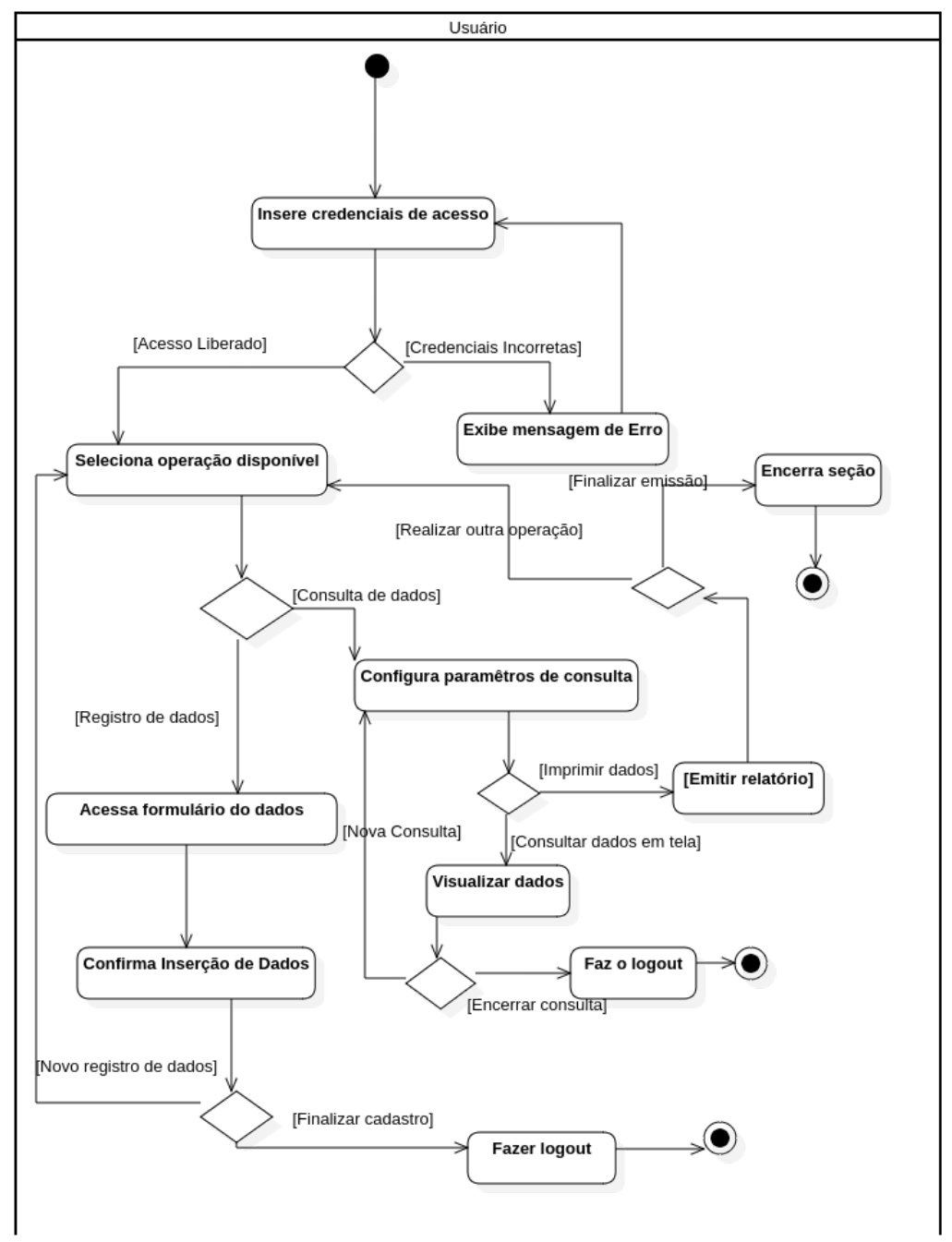

Figura 5. Diagrama de Atividade - Fluxo Típico no Sistema

\subsection{Funcionalidades da ferramenta}

As principais funcionalidades da ferramenta são a seguir apresentadas. O cadastro das informações está alinhado com os protocolos seguidos pela equipe que realiza o monitoramento do vírus da febre amarela. A Figura 6 mostra a tela de cadastro das informações coletadas nas visitas de campo. Observe que é possível selecionar a atividade em que ocorreu a implantação da armadilha. A amostra colhida recebe um identificador e suas coordenadas geográficas são obtidas do GPS do pesquisador de campo. São informados os dados coletados na armadilha, com a numeração de larvas e de pupas e observando as várias alturas de coleta estipuladas para cada armadilha. Posteriormente, o pesquisador de laboratório cataloga o número de ovos. 


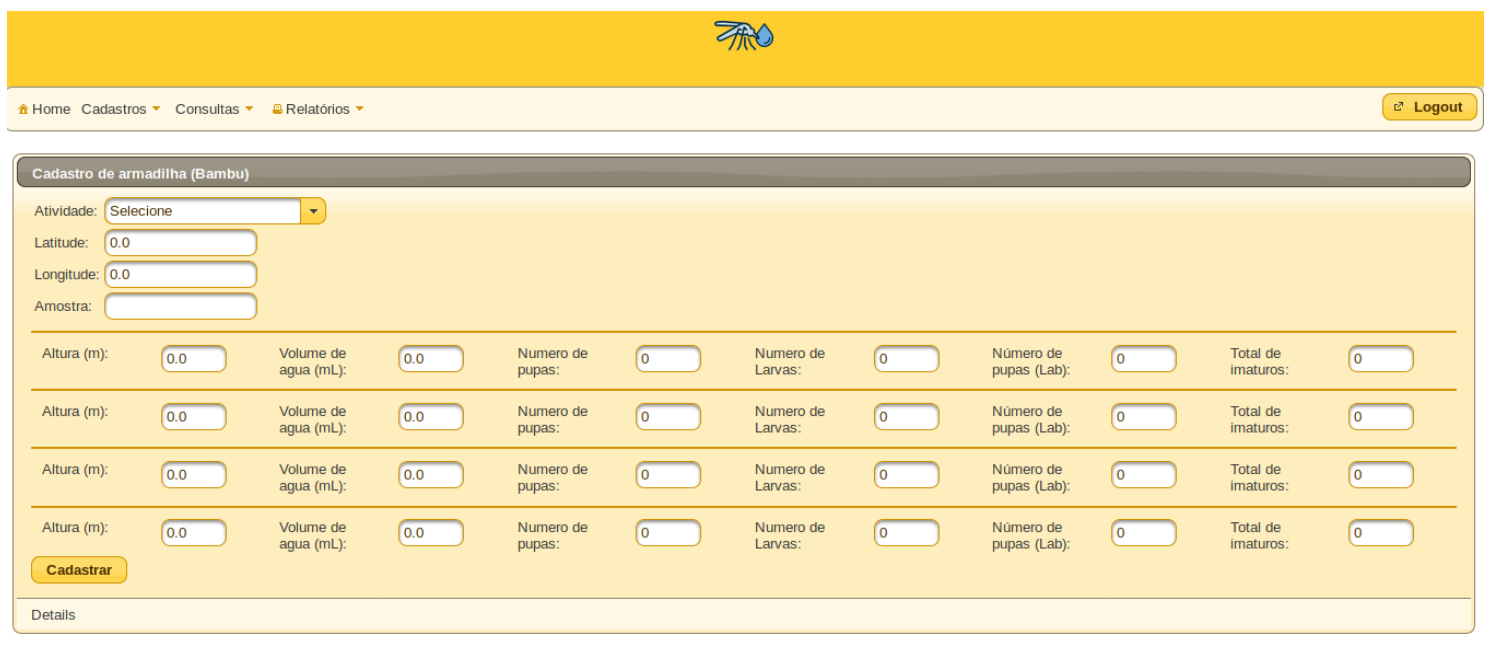

Figura 6. Cadastro de Armadilhas

Um mapa dinâmico, exibido na Figura 7, tornou a visualização de armadilhas mais intuitiva. O mapa utiliza a Interface de Programação de Aplicação (em inglês, Appplication Programming Interface (API)) do ArcGIS JavaScript implementado pelo GISFaces, e usa dados das armadilhas Ovitrampas e Bambus registradas nas áreas de estudo elencadas. A visualização dos dados referentes à Unidade de Conservação retorna os dados das últimas atividades executadas e da situação das armadilhas implantadas em cada área. As armadilhas dispostas em vermelho são Bambus e em amarelo são Ovitrampas.

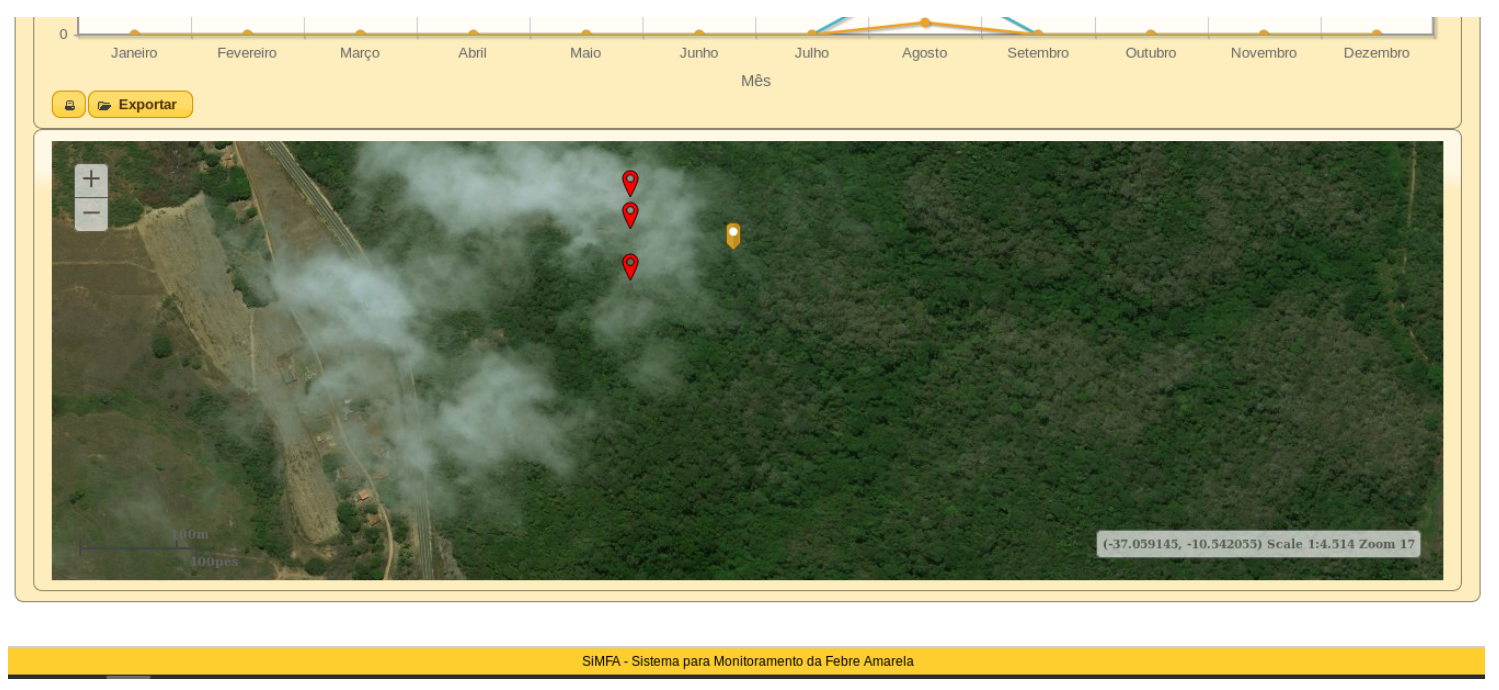

Figura 7. Mapa de Armadilhas

A ferramenta conta com funcionalidade para emissão de relatórios em formato PDF, permitindo configurar relatórios das armadilhas registradas e gerar os relatórios. Na Figura 8 é apresentado um exemplo de relatório geral de ovitrampas emitido pela ferramenta, onde são dispostos os dados de todas as armadilhas ovitrampas implantadas, com informações de localidade, altura, números das amostras coletadas e datas de imersão e eclosão dos ovos. 


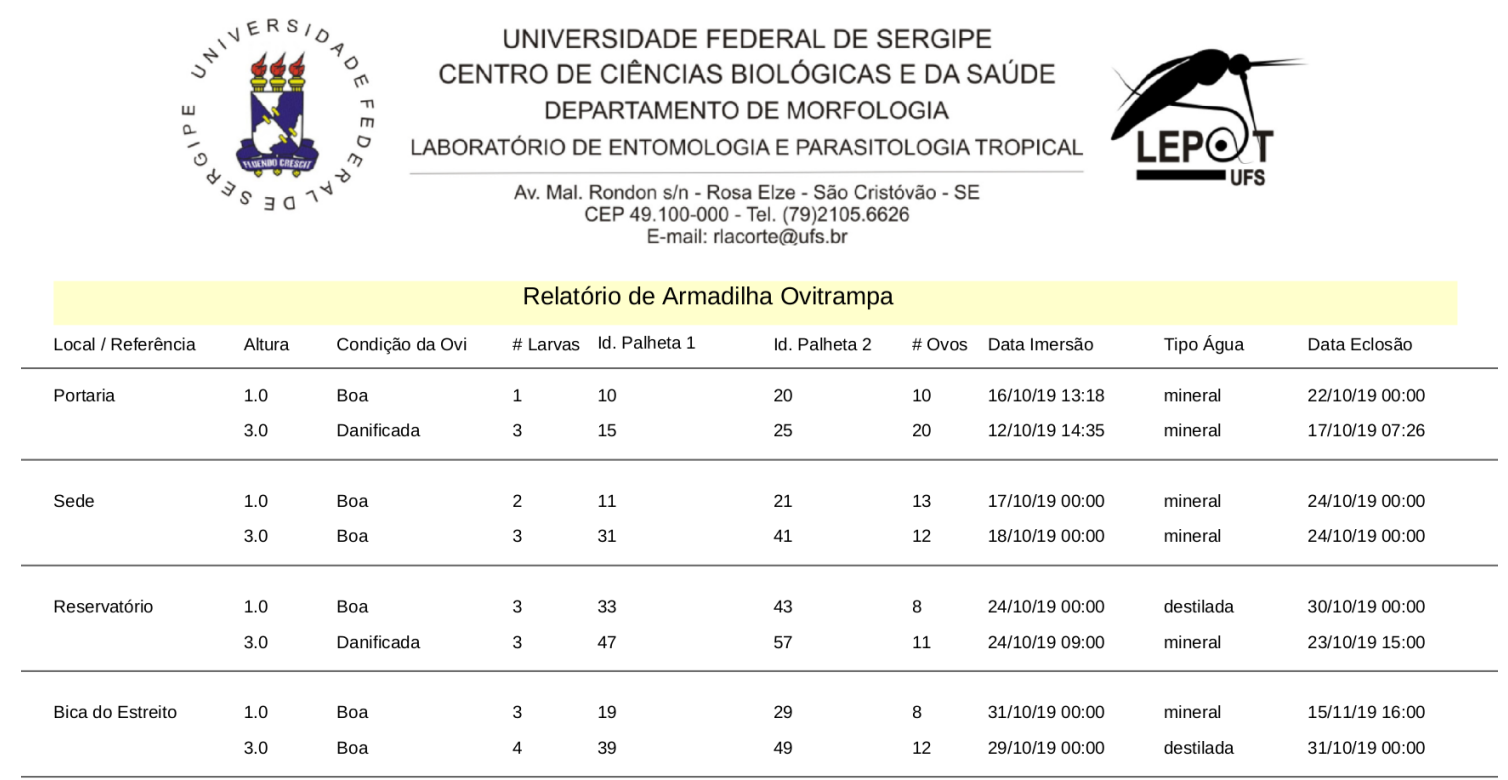

Figura 8. Relatório de Armadilhas

A funcionalidade de exportar dados também está contemplada no sistema, possibilitando a exportação das informações de atividades executadas nas unidades de conservação no formato escolhido, sendo disponíveis os formatos: XLS e CSV. Ainda na Figura 9 é apresentada uma tabela com as atividades executadas, local de execução e o período, assim como um gráfico com o histórico de armadilhas no ano determinado. Os dados referentes às tabelas podem ser exportados para os formatos previstos.

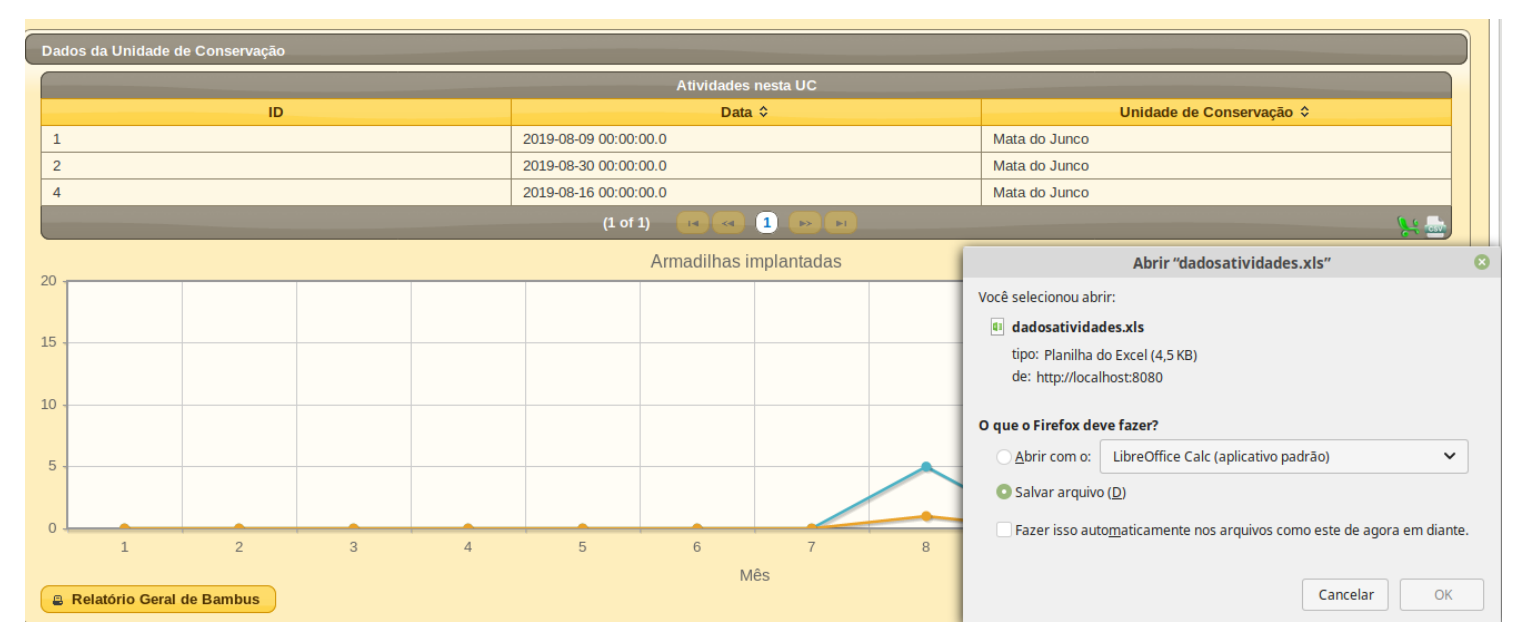

Figura 9. Exportação de dados

O sistema conta com uma aplicação para dispositivos móveis, para possibilitar que os pesquisadores preencham as informações de coleta durante a execução de atividades nas unidades de conservação. A Figura 10 mostra, na tela à esquerda, a tela principal do sistema e na tela à direita, o mesmo cadastro que o exibido na Figura 6. 


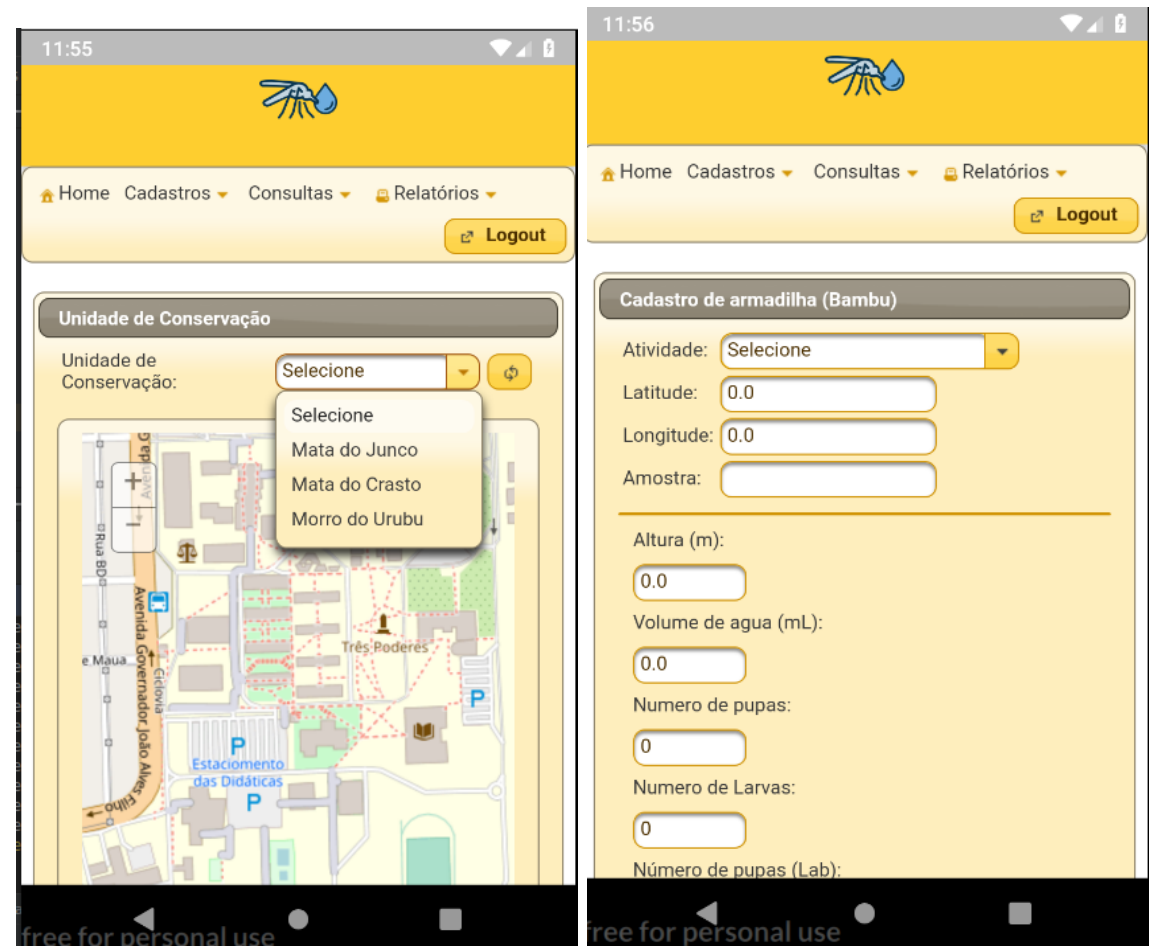

Figura 10. Tela de visualização em dispositivo móvel

\section{Conclusão}

Este trabalho objetivou o desenvolvimento de uma ferramenta para dar suporte no monitoramento e controle da febre amarela. A solução provê apoio computadorizado às atividades realizadas em pesquisas de campo e laboratoriais. Os dados obtidos para validação da ferramenta foram coletados nas matas presentes nas áreas suscetíveis, que estão sendo monitoradas pelos pesquisadores. Como trabalhos futuros imediatos, pretende-se desenvolver um módulo que dê apoio às atividades de pesquisa documental, não contempladas nesta versão da ferramenta, bem como a validação extensiva da ferramenta desenvolvida com dados coletados no decorrer do projeto.

Pelo fato do desenvolvimento do sistema ter ocorrido em paralelo à realização do projeto de monitoramento das áreas suscetíveis, a validação foi realizada com uma amostra limitada de dados atualizados. Assim, para complementar as informações de validação foram utilizados dados históricos. Com a evolução do projeto, mais dados serão coletados enriquecendo o sistema com mais informações. Também serão aplicados testes de usabilidade junto aos pesquisadores da área de saúde envolvidos no projeto.

Com o emprego efetivo da ferramenta, junto às atividades da equipe de monitoramento, espera-se como resultado um avanço na gestão e apresentação dos dados epidemiológicos relativos à febre amarela. Em casos de ressurgência dos surtos nas regiões, as atividades de monitoramento, incluindo aí o uso do sistema, podem fazer previsões, possibilitando alertar os órgãos de saúde competentes para a tomada de ações, evitando maiores danos à saúde da população. Com o uso e futuras adequações do sistema decorrentes do amadurecimento das atividades de monitoramento realizadas no âmbito deste projeto, o sistema aqui proposto poderá se consolidar nos programas de controle promovidos pelo SUS. 


\section{Referências}

Consoli, R. and Lourenço-de Oliveira, R. (1994). Principais mosquitos de importância sanitária no Brasil. Editora FIOCRUZ, Rio de Janeiro, RJ, BRA.

Correa, R. and Ramalho, G. (1956). Revisão de phoniomyia theobald, 1903 (diptera, culicidae, sabethini). Folia Clinica et Biologica, 25(1).

Dialynas, E., Topalis, P., Vontas, J., and Louis, C. (2009). Miro and irbase: It tools for the epidemiological monitoring of insecticide resistance in mosquito disease vectors. PLoS Neglected Tropical Diseases, 3(6) art. no. e465.

FIOCRUZ (2015). Sistema de alerta: Infodengue disponível em https://info.dengue.mat.br/.

Forattini, O. (1965). Entomologia médica: Culicini: Culex, Aedes e Psorophora. Editora da Universidade de São Paulo, São Paulo, SP, BRA.

Forattini, O. (2002). Culicidologia médica: identificação, biologia, epidemiologia. Editora da Universidade de São Paulo, São Paulo, SP, BRA.

Fowler, M. (2005). UML Essencial 3ed. Bookman, Porto Alegre, RS, BRA.

Gradella, G., Padilha, J., Zanatta, E., Portella, F., Oliveira, A., Eugênia, M., Dahmer, A., Junior, J., and Tubelo, R. (2016). O design da informação aplicado ao desenvolvimento da interface gráfica de um jogo sério sobre o combate ao mosquito aedes aegypti. Jornal Brasileiro de Telessaúde, 4:246-254.

Lane, J. and Cerqueira, N. (1942). Os Sabetineos da América, (Diptera, Culicidae). Arquivos de zoologia do estado de Sao Paulo. Imprensa oficial do estado.

Morais, H. S., Santos, O. S., Rocha, M. A., Almeida, T. C. S., Brasil, L. M., Amvame-Nze, G. D., Miosso, C. J., Costa, M. V. C., and Pizo, G. A. (2015). Monitoring information system of aedes aegypti reproduction. In Jaffray, D. A., editor, World Congress on Medical Physics and Biomedical Engineering, June 7-12, 2015, Toronto, Canada, pages 1423-1426, Cham. Springer International Publishing.

MS (1998). Ministério saúde sistema de informação de agravos de notificação (SINAN) disponível em http://www.saude.gov.br/sinan_net.

MS (2017). Informe n 28/2017 - Monitoramento dos casos e óbitos de febre amarela no Brasil. Centro de Operações de Emergências em Saúde Pública Sobre Febre Amarela, Brasília, BRA.

OMS (2019). Relatório Organização Mundial da Saúde disponível em https://www.who.int/en/news-room/fact-sheets/detail/yellow-fever.

Reinert, J. (2001). Revised list of abbreviations for genera and subgenera of culicidae (diptera) and notes on generic and subgeneric changes. Journal of the American Mosquito Control Association, 17. 